\section{Studienzentrum der Deutschen Gesellschaft für Chirurgie (SDGC): Bessere Chirurgische Versorgung in Deutschland durch Förderung klinischer Studien}

Die Deutsche Gesellschaft für Chirurgie (DGCh) hat ein Studienzentrum speziell für die Chirurgie eingerichtet. Sie fördert damit die Planung, Durchführung und Auswertung großer multizentrischer Studien. Mit dem Studienzentrum der Deutschen Gesellschaft für Chirurgie (SDGC) stärkt die Fachgesellschaft die patientenorientierte Forschung gegenüber der Grundlagenforschung. Das SDGC hat vor allem die Aufgabe, die chirurgische Versorgung in Deutschland zu verbessern.

In den letzten Jahren ist ein Mangel an guten klinischen Studien in der Chirurgie - auch im internationalen Vergleich - zunehmend deutlich geworden. Um sich jedoch weiter zu entwickeln, braucht die Chirurgie verlässliche Ergebnisse aus der klinischen Forschung. Professor Dr. med. Hartwig Bauer, Generalsekretär der DGCh, betont: «Die hohen wissenschaftlichen, methodischen und ethischen Anforderungen an diese Studien bedingen die Notwendigkeit einer speziellen Einrichtung.»

Chirurgische Studien unterscheiden sich grundsätzlich von Studien mit Arznei- mitteln: «Besondere Herausforderungen sind die Standardisierung und Reproduzierbarkeit von chirurgischen Eingriffen» betont Dr. med. Christoph Seiler von der Chirurgischen Klinik der Universität Heidelberg, Chirurg und Klinischer Epidemiologe, der das SDGC leitet.

Die derzeit größte vom SDGC geführte Studie untersucht den «Bauchdeckenverschluss»: Bei etwa zehn Prozent der Patienten bricht nach einer Operation in der Bauchhöhle die Bauchwand entlang der Narbe erneut auf. Die Behandlung von Bauchwandbrüchen ist bei dieser Häufigkeit auch von großer sozioökonomischer Bedeutung. An mehr als 600 Patienten untersuchen Chirurgen deshalb jetzt, wie sich die Bauchdecke so verschließen lässt, dass das Risiko späterer Narbenbrüche deutlich reduziert wird, erläutert Dr. Seiler. Insgesamt 20 Kliniken aller Versorgungsstufen in ganz Deutschland - von der Universität bis zur Grund- und Regelversorgung beteiligen sich an dieser Studie. Mit ersten Ergebnissen rechnet das SDGC im Frühjahr 2007. Weitere Studien auf unterschiedlichen Gebieten der Chirurgie laufen bereits oder sind geplant.

Das Bundesministerium für Bildung und Forschung (BMBF) fördert das Studienzentrum seit dem 1. Januar $2005 \mathrm{im}$ Rahmen des Strukturprogramms zur
Verbesserung der patientenorientierten Forschung in Deutschland. Das SDGC ist außerordentliches Mitglied der Koordinierungszentren für klinische Studien (KKS). Mit weiteren Institutionen im In- und Ausland, zum Beispiel der American College of Surgeons Oncology Group, ist es engverbunden.

Das Studienzentrum der DGCh unterstützt die nationale Initiative zur Studienregistrierung. Alle Studien des SDGC sind bereits international registriert. Zusammen mit dem KKS-Netzwerk führt das SDGC ein umfangreiches Aus- und Weiterbildungsprogramm für Chirurgen und Pflegekräfte für die sachgerechte Planung und Durchführung von Studien in der Chirurgie durch.

Für die künftige Entwicklung der Deutschen Chirurgie ist das SDGC von groBer Bedeutung. Die Deutsche Gesellschaft für Chirurgie hat gemeinsam mit ihren assoziierten chirurgisch-wissenschaftlichen Fachgesellschaften mit dem SDGC eine Einrichtung geschaffen, die derzeit einzigartig in der Landschaft der wissenschaftlichen Fachgesellschaften ist.

Weitere Informationen bei Deutsche Gesellschaft für Chirurgie Pressestelle

Tel. +49 711 8931-552, Fax -167

info@medizinkommunikation.org

\title{
Ticker+++ Ticker+++ Ticker+++ Ticker+++ Ticker+++ Ticker+++ Ticker+++
}

Wyeth Pharama GmbH. Das neue Antibiotikum Tygacil ${ }^{\circledR}$ (Tigecyclin) wurde von der amerikanischen Gesundheitsbehörde FDA (Food and Drug Administration) zur Therapie von schweren intraabdominellen Infektionen sowie von komplizierten Infektionen der Haut und Weichteile zugelassen.

Weitere Informationen be

Medizin \& Markt, Dr. Birte Friess

Tel. +49 89 3-839300, Fax -39075

info@medizin-und-markt.de
Pfrimmer Nutricia GmbH. Für seine Untersuchung über die Wirkungsweise von Hormonen bei der Regulierung der menschlichen Nahrungsaufnahme erhielt Dr. Jean-Pierre Gutzwiller aus Basel den diesjährigen Dr.-Werner-FeklFörderpreis für klinische Ernährung. Weitere Informationen bei BirkelPartner GmbH, Meike Hallbauer Tel. +49 9131 8842-16, Fax -44 meike.hallbauer@birke.de
Abbott GmbH. Eine Phase-III-Studie, die bei der diesjährigen Digestive Disease Week (14. 19. Mai 2005) vorgestellt wurde, zeigt, dass fast $80 \%$ der Morbus-Crohn-Patienten auf Humira ${ }^{\circledR}$ (Adalimumab) ansprechen. Ein Drittel der $\mathrm{Pa}$ tienten unter Humira erreichte nach 24 Wochen eine klinische Remission.

Weitere Informationen bei

Weber Shandwick, Susanne Buder

Tel. +49 69913 043-23, Fax -559 sbuder@webershandwick.com

\begin{tabular}{ll}
\hline KARGER & ( 2005 S. Karger GmbH, Freiburg \\
Fax +497614520714 & $\begin{array}{l}\text { Accessible online at: } \\
\text { www.karger.com/cga }\end{array}$
\end{tabular}

